decisions of the treating physician. Different therapeutic strategies are adopted regarding the treatment, and in many cases highly expensive drugs as Ivlg are prescribed. Further studies and recommendations are certainly needed for homogenising the patient management for this severe aspect of the disease. Disclosure of Interest: None declared

DOI: 10.1136/annrheumdis-2018-eular.4261

\section{AB0807 PLATELET INDICES AS MARKERS OF INFLAMMATION IN SYSTEMIC SCLEROSIS}

S. Eldessouki lbrahim ${ }^{1}$, C. Morad ${ }^{2} .{ }^{1}$ Ain shams University, Cairo, Egypt, ${ }^{2}$ Internal medicine, Ain shams University, Cairo, Egypt

Background: Systemic sclerosis (SSc) is a connective tissue disease involving multiple organs with an unknown etiology. Platelet function may be associated with endothelial dysfunction and immune regulatory mechanisms. Recently, an increased tendency to platelet aggregation and enhanced platelet activation have been described in SSc patients, suggesting a role for platelets in the disease itself. $^{12}$

Objectives: To evaluate platelet indices in systemic sclerosis (SSc) patients and identify their clinical significance as novel inflammatory biomarkers in correlation to markers of endothelial dysfunction: vascular endothelial growth factor (VEGF) and flow mediated dilatation (FMD).

Methods: Thirty-five SSc patients were enrolled in addition to thirty-five age and sex matched healthy volunteers as controls. All patients and controls underwent full medical history taking, thorough clinical examination, assessment of severity extent of skin sclerosis using the modified Rodnan skin score, erythrocyte sedimentation rate (ESR), C- reactive protein (CRP), complete blood count with special consideration to mean platelet volume (MPV), platelet distribution width and platelets count, assay for serum VEGF concentration, and brachial FMD assessment by colour duplex sonography.

Results: There was a highly significant decrease in the mean MPV in SSc patients compared to the controls $(8.65 \pm 0.6 \mathrm{fl}$ vs. $9.55 \pm 0.52 \mathrm{fl})$. There was a significant increase in the mean platelet count in SSc patients compared to controls $\left(331.63 \pm 64.66 \times 10^{3} / \mathrm{ml}\right.$ vs. $\left.297.80 \pm 44.48 \times 10^{3} / \mathrm{ml}\right)$. In SSc patients, a significant negative correlation was found between the mean MPV and each of ESR, CRP and VEGF ( $r=-0.42,-0.368$ and -0.55 respectively, $p<0.05)$; and a significant positive correlation was found between the mean MPV and mean FMD ( $r=0.378$, $\mathrm{p}<0.05)$. Linear regression test, showed an association between mean MPV and each of ESR and CRP ( $t=-3.312,-2.92$ respectively, $\mathrm{p}<0.05)$.

Conclusions: MPV levels could be an easily measurable parameter to reflect the inflammatory condition and disease activity in systemic sclerosis patients.

\section{REFERENCES:}

[1] da Silva IF, Teixeira A, Oliveira J, Almeida R, Vasconcelos C 2017 Jan 27

[2] Manetti M The crowded crossroad to angiogenesis in systemic sclerosis: where is the key to the problem? Arthritis Res Ther 2016;18:36

Disclosure of Interest: None declared

DOI: 10.1136/annrheumdis-2018-eular.1152

\section{AB0808 IN TUNISIA}

S. Hammami ${ }^{1}$, S. Bouomrani ${ }^{2}$, O. Berriche ${ }^{3}$, A. Barhoumi ${ }^{4}$, on behalf of The study Group for Scleroderma, STMI. 'Department of Internal Medicine, CHU F Bourguiba Monastir, Monastir, ${ }^{2}$ Internal medicine, Military hospital Gabes, Gabes; ${ }^{3}$ Department of Internal Medicine, CHU T Sfar Mahdia, Mahdia; ${ }^{4} \mathrm{CHU} F$ Bourguiba, University of Monastir, Monastir, Tunisia

Background: Scleroderma is an autoimmune connective tissue disorder which is characterised by fibrosis of visceral organs, skin and blood vessels. This condition can be localised or systemic. Its estimated prevalence is 250 cases in a million and it is more common in women than in men.

Objectives: The aim of this retrospective multicenter study was to analyse demographic, clinical, laboratory features and outcome of SSc in Tunisia throughout 8 Departments of Internal Medicine and to compare them with those of other geographic groups.

Methods: One hundred and eight cases of SSc were recorded (American College of Rheumatology criteria) during a 15 years period. They were 93 women and 15 men with an average age at SSc onset of approximately 46.9 years. ${ }^{17-75}$

Results: Only 18 patients had limited cutaneous SSc, 11 patients had a CREST syndrome. Our Tunisian patients were characterised by a high frequency of cutaneous signs: sclerodactily $(80.6 \%)$, proximal sclerosis $(81 \%)$, telangiectasia $(34.3 \%)$, Raynaud's syndrome (91\%), pigmentation disorders (36\%), ulceration $(24 \%)$ and subcutaneous calcification (4.6\%). The other clinical manifestations were dysphagia in $54 \%(n=59)$, pulmonary involvement in $55.6 \%(n=60)$, cardiac manifestation in $27 \%(n=29)$, arthritis in $19.4 \%(n=21)$, renal involvement in $13.8 \%$ $(n=15)$, and neurological involvement in 12 cas. $78.7 \%$ were antinuclear antibody (ANA) positive, 25\% were Antitopoisomerase-I antibodies (anti-Scl-70 antibody) positive and $10 \%$ with anti-centromere antibodies.

SSc was associated to Sjögren's syndrome $n=27$, systemic lupus erythematosus $(\mathrm{n}=8)$, Polymyositis $(\mathrm{n}=5)$ and Rheumatoid arthritis $(\mathrm{n}=1)$

Treatment regimen included, calcium channel blockers $(65.7 \%)$, steroids (47\%) Colchicine $(47.2 \%)$, D penicillamine $(21 \%)$, immuosuppressive therapy was added in patient with partial control $(n=13)$. Median follow-up period was 3.7 years. With the above treatment protocol, $(29.6 \%)$ patients achieved disease control on treatment, $(43.3 \%)$ had partial control while $(26.8 \%)$ showed no response or progressive disease, Six patients died, three of them with scleroderma renal crisis.

Conclusions: The findings of this study documents The high frequency of exten sive cutaneous sclerosis, Potential limitations and biases in our study need discussion. Specific recruitment of patients in tertiary referral centres may be the source of selection bias. Patients were evaluated by different doctors. The therapeutic management and outcome monitoring were heterogeneous. This study remains the most representative of Tunisian Scleroderma patients recruited from all parts of Tunisia

\section{REFERENCES:}

[1] Rongioletti F, Ferreli C, Atzori L, Bottoni U, Soda G. Scleroderma with an update about clinico-pathological correlation.G Ital Dermatol Venereol. 2018 Jan 24

[] 2

Diao M, Ndiaye MB, Kane A, Bodian M, Tchintchui NC, Mbaye A, Mounir Dia M, Sarr M, Kane A, Abdou Ba S. Pulmonary hypertension in scleroderma: about 12 cases. Pan Afr Med J. 2012;11:9. Epub 2012 Jan 18

Acknowledgements: Al the group of the scleroderma Study STMI

Disclosure of Interest: None declared

DOI: 10.1136/annrheumdis-2018-eular.6247

\section{AB0809 \\ A NEW SCORE TO PREDICT DIGITAL ULCERS COMBINING CLINICAL DATA, IMAGING AND PATIENT HISTORY IN SYSTEMIC SCLEROSIS}

S. Friedrich $^{1,2}$, S. Lüders ${ }^{1,3}$, G.-R. Burmester ${ }^{1}$, G. Riemekasten ${ }^{4}$, S. Ohrndorf ${ }^{1}$

${ }^{1}$ Department of Rheumatology and Clinical Immunology, ${ }^{2}$ Department of

Radiology; ${ }^{3}$ Department of Gastroenterology and Rheumatology, Charite Universtätsmedizin Berlin, Berlin; ${ }^{4}$ Department of Rheumatology and Clinical Immunology, University of Schleswig-Holstein, Lübeck, Germany

Background: Ischaemic complications such as digital ulcers (DU) are a common complication in systemic sclerosis (SSc) patients.

Objectives: The aim of this study was to combine clinical characteristics and imaging methods to a composite predictive score.

Methods: Seventy-nine SSc patients received clinical examination and their patient history was taken. Furthermore, we performed nailfold capillaroscopy (NC), colour Doppler ultrasonography (CDUS) and fluorescence optical imaging (FOI) of the hands at baseline. Newly developed digital ulcers over a period of approximately 12 months were registered. We used criteria with significant $(p<0.5)$ OR values above 3.5 in regard to the development of these new DU to create the score (CIP-DUS, clinical features, imaging, patient history - digital ulcer score)

Results: Twenty-nine percent of the patients developed new DU during follow-up (48.1\% diffuse SSc, $18.4 \%$ limited SSc). The following criteria were used: SSc diffuse subtype (OR 4.127, $p=0.0087$ ), modified Rodnan skin score $>8$ (OR 9.429 [95\% $\%$ : 3.0-29.2], $\mathrm{p}<0.0001$ ), pulmonary arterial hypertension (OR 6.854 [95\% $\mathrm{Cl}$ : 1.6-9.7], $\mathrm{p}=0.0088$ ), present digital ulcers or pitting scars at baseline (OR 15.71 [95\% Cl: $3.3-74.3], \mathrm{p}<0.0001$ ), history of digital ulcer or pitting scars (OR 36.15 [95\%Cl: 2.1-626.9], p<0.0001), NC pattern (OR 18.6 [95\%Cl: 1.1-326.4], $\mathrm{p}=0.0035)$, reduced capillary density $(\mathrm{n}<7 / \mathrm{mm})$ in digit III of the right hand in NC (OR 9.0 [95\% $\%$ : 1.1-73.6], $\mathrm{p}=0.0266$ ), missing initial enhancement in FOl in digit III of the right hand (OR 3.857 [95\% $\%$ : $1.2-12.8], p=0.0323$ ), percentage of pathologic (i. e. narrowed or occluded) vessels $>35 \%$ in CDUS (OR 4.286 [95\% Cl: $1.5-$ 12.4], $p=0.0099)$. Criteria with greater OR should impact the score to a higher degree so we appointed three points to dichotomous criteria with $\mathrm{OR}>10$, two points for criteria with OR between $5-10$, and one point for criteria with $\mathrm{OR}<5$. Regarding the NC pattern, 3 points were given to patients with late pattern, 2 points for active and 1 point for early pattern.

Best results were found for a cut-off of $>10$ points with obtained sensitivity levels of $95 \%$ and specificity levels of $74 \%$ in regard to new DU ( $A U C=0.8687$, $\mathrm{p}<0.0001)$. In the absence of CDUS and FOI data, specificity levels dropped slightly to $72 \%$ with unchanged sensitivity values of $95 \%$. 